\title{
Utilizing the Natural Composition of Brown Seaweed for the Preparation of Hybrid Ink for 3D Printing of Hydrogels
}

\author{
Linn Berglund,* Jonathan Rakar, Johan P. E. Junker, Fredrik Forsberg, and Kristiina Oksman*
}

Cite This: ACS Appl. Bio Mater. 2020, 3, 6510-6520

Read Online

ABSTRACT: This study aims to utilize the natural composition of brown seaweed by deriving alginate and cellulose concurrently from the stipe (stem-like) and blade (leaf-like) structures of the seaweed; further, this is followed by fibrillation for the direct and resource-efficient preparation of alginate/cellulose nanofiber (CNF) hybrid inks for three-dimensional (3D) printing of hydrogels. The efficiency of the fibrillation process was evaluated, and the obtained gels were further studied with regard to their rheological behavior. As a proof of concept, the inks were $3 \mathrm{D}$ printed into discs, followed by cross-linking with $\mathrm{CaCl}_{2}$ to form biomimetic hydrogels. It was shown that the nanofibrillation process from both seaweed structures is very energy-efficient, with an energy demand lower than 1.5 $\mathrm{kW} \mathrm{h} / \mathrm{kg}$, and with CNF dimensions below $15 \mathrm{~nm}$. The inks displayed excellent shear-thinning behavior and cytocompatibility and were

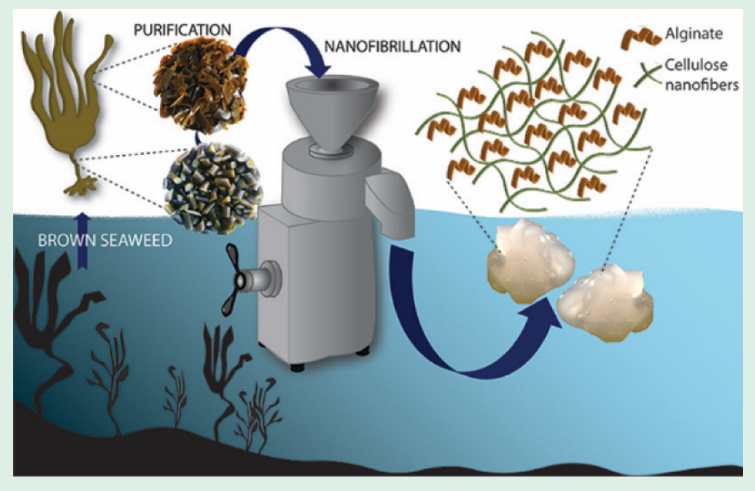
successfully printed into 3D discs that, after cross-linking, exhibited an interconnected network structure with favorable mechanical properties, and a cell viability of $71 \%$. The designed $3 \mathrm{D}$ biomimetic hydrogels offers an environmentally benign, cost-efficient, and biocompatible material platform with a favorable structure for the development of biomedical devices, such as 3D bio printing of soft tissues.

KEYWORDS: alginate, cellulose nanofibers, 3D printing, biomimetic hydrogels, biomedical application, tissue engineering

\section{INTRODUCTION}

Brown seaweed is an encouraging and fascinating natural resource with increasing commercial and ecological importance. ${ }^{1}$ Laminaria digitata is one type of fast-growing and large brown seaweed, which consists of a holdfast (root-like), a stipe (stem-like), and a blade (leaf-like) structure that can grow up to $4 \mathrm{~m}$ in length. ${ }^{2}$ It grows along the coasts in the relatively cold Northern hemisphere, and its carbohydrate composition varies with season, geographic location, and age, ${ }^{3}$ as well as between the different parts of the seaweed (stipe and blade). ${ }^{4}$ The polysaccharides in brown seaweed differ considerably from those found in terrestrial plants; although cellulose is present in smaller amounts, alginate is the major structural component of the cell wall. Thus, the most common source of alginate for commercial extraction is brown seaweed, ${ }^{2}$ which has further industrial uses such as in dental impression and wound dressing materials and as a stabilizer and thickener in ice cream and food.

Alginate consists of 1,4-glycosidically linked $\alpha$-L guluronic acid $(\mathrm{G})$ and $\beta$-D-mannuronic acid (M). The linear chains are made up of different blocks of guluronic and mannuronic acids, referred to as MM or GG blocks (MG or GM blocks), where the linkage in the block structure results in varying degrees of flexibility or stiffness in the alginates. In the presence of $\mathrm{Ca}^{2+}$, the GG blocks form ionic complexes to generate a stacked (cross-linked) structure known as the "egg-box model", which is responsible for strong gel formation. ${ }^{6}$ Combined with its biodegradability, biocompatibility, nonantigenicity, and chelating ability, ${ }^{5}$ alginate has been widely utilized in the assembly of hydrogels for biomedical applications such as tissue engineering of soft $^{7,8}$ - and bone ${ }^{9}$-tissue, in addition to wound healing ${ }^{8}$ and drug delivery. ${ }^{10}$ Three-dimensional (3D) printing of alginate has triggered increased attention to the assembly of hydrogels for biomedical purposes, where one of the main challenges lies in achieving shape fidelity of the $3 \mathrm{D}$ structure. Although the viscosity of alginate can be adjusted through its concentration and molecular weight, ${ }^{11}$ its rheological behavior is not sufficient for structural integrity while printing. Several researchers have solved this problem by introducing cellulose nanofibers (CNFs) as a second component to tailor commercial alginate as ink suitable for $3 \mathrm{D}$ printing.,

CNFs are attractive for biomedical applications owing to their good mechanical properties and biocompatibility, ${ }^{13}$

Received: July 24, 2020

Accepted: August 29, 2020

Published: August 29, 2020 
where a reinforcing effect by significantly enhancing the compressive properties of alginate hydrogels has been reported. ${ }^{9}$ In another study, ${ }^{14} \mathrm{CNF}$ were shown to be beneficial for the dimension stability and mechanical properties of alginate-based hydrogels, and the presence of an entangled nanofiber network was observed to affect the pore structures, enhancing their size and thus making them more suitable for cell growth.

For 3D printing, the introduction of CNFs has contributed with a shear-thinning behavior that have enabled the printing of $3 \mathrm{D}$ shapes. $^{7}$ However, the $3 \mathrm{D}$ printing process requires optimization of the alginate/CNF ratio. Their synergistic behavior and essential relative proportion is further dictated by the characteristics of both the alginates and CNFs, which in turn is connected to how the different components have been isolated. ${ }^{5,12}$ The ratio must therefore be optimized for each material formulation to achieve printability. From a sustainability and efficiency perspective, extensive separation processing steps are required to derive these two components from biomass; ${ }^{13,15,16}$ in contrast, both alginate and cellulose are naturally found in brown seaweed. ${ }^{6}$

Although there are previous reports on the printability of alginate/CNF combinations from biomass, ${ }^{7,9,17,18}$ the use of seaweed's natural composition to achieve a multifunctional gel suitable for 3D printing has not been in focus.

Inspired by a biomimetic approach-the natural design of the cell wall structure-we aim to utilize the natural composition of alginate and cellulose found in brown seaweed by deriving them together from both the stipe (stem-like) and blade (leaf-like) parts. Followed by fibrillation, this is a resource-efficient preparation of alginate/CNF-based hybrid inks. In the preparation of the hybrid inks from the stipe and blade, the energy demand, degree of fibrillation, obtained nanostructures, and natural composition were evaluated and compared. The cross-linking ability of alginate and the shearthinning behavior of CNF was assessed through rheology measurements, before printing into $3 \mathrm{D}$ discs, followed by cross-linking with $\mathrm{CaCl}_{2}$ to form biomimetic hydrogels. The $3 \mathrm{D}$ structure, compressive properties, and cytocompatibility of the hydrogels were finally studied to evaluate their suitability for biomedical applications.

\section{EXPERIMENTAL SECTION}

Materials. Brown seaweed (L. digitata) was kindly provided by The Northern Company Co. (Træna, Norway) and used as the raw material in this study. The contents of the raw material are reported in Supporting Information (Table S1). The seaweed was harvested on the west coast of the North Atlantic Ocean in May 2017. The fresh samples were stored in closed bags in the freezer before use. The stipe and blade of the seaweed were prepared in separate batches for comparison and utilization of the entire structure. Both materials were purified and nanofibrillated using equivalent processing conditions.

Sodium chlorite $\left(\mathrm{NaClO}_{2}\right)$, sodium hydroxide (pure pellets, $\mathrm{NaOH})$, and acetic acid $\left(96 \% \mathrm{CH}_{3} \mathrm{COOH}\right)$ were purchased from Merck KGaA, Darmstadt, Germany. For ionic cross-linking, calcium chloride of laboratory grade was used $\left(\mathrm{CaCl}_{2} \cdot 2 \mathrm{H}_{2} \mathrm{O}, 90 \mathrm{mM}\right.$; from Sigma-Aldrich Sweden AB, Stockholm, Sweden). All chemicals were used as received. Deionized water was used for all experiments.

Direct Preparation of Hybrid Inks. The stipe and blade of the seaweed were left in room temperature $\left(22 \pm 1{ }^{\circ} \mathrm{C}\right)$ for about $24 \mathrm{~h}$ to defrost; subsequently these were cut into smaller pieces prior to purification by bleaching with $\mathrm{NaClO}_{2}(1.7 \%)$ in an acetate buffer $(\mathrm{pH} 4.5)$ at $80{ }^{\circ} \mathrm{C}$ for $2 \mathrm{~h}$. After purification, all color was removed and the material was washed until a neutral $\mathrm{pH}$ was reached. The solid recovery was calculated as a yield according to the following equation

$$
\text { yield }(\%)=W_{1} / W_{0} \times 100
$$

where $W_{1}$ indicates the dry weight of the sample after the purification and $W_{0}$ denotes the initial dry weight of the seaweed. The presented yields are based on the average of three different batches of each material.

The materials (at a concentration of 2 wt \%) were nanofibrillated using an MKZA6-3 ultrafine friction grinder, Masuko Sangyo Co., Ltd. (Kawaguchi, Japan) with coarse silica carbide $(\mathrm{SiC})$ grinding stones. The structures were mostly intact after the purification, so the first pass through the Masuko grinder was performed with an open gap $(20 \mu \mathrm{m})$ to predisperse the materials, given that no other dispersing equipment was used. The nanofibrillation was conducted in contact mode, with the gap of the two discs set to $-90 \mu \mathrm{m}$, at 1500 rpm. The prepared hybrid inks were denoted S-A-CNF (stipealginate-CNFs) and B-A-CNF (blade-alginate-CNFs).

The energy consumption of the fibrillation process was established by the direct measurement of the power with an energy analyzer, EM24 DIN, Carlo Gavazzi (Belluno, Italy) and from the monitored processing time. The energy demand was calculated from the product of the power and the time, and the energy consumption is expressed as kilowatt-hour per kilogram of dry weight of nanofiber. The first sample was collected after the first pass through the Masuko grinder with an open gap. Samples were subsequently collected at regular intervals to assess the degree of fibrillation. The process was finalized when a plateau was reached in the viscosity and no larger structures could be observed by microscope. The prepared inks were degassed and kept in a refrigerator at $6{ }^{\circ} \mathrm{C}$ prior to the $3 \mathrm{D}$ printing of the hydrogels. To further study the nanofibers and their network formation, films were prepared for tensile testing using the solvent casting method, where the dispersions were diluted to $1 \mathrm{wt} \%$ concentration by adding water under magnetic stirring, degassed, and subsequently left to dry in Petri dishes for about 3 day at room temperature $\left(22.6 \pm 2{ }^{\circ} \mathrm{C}\right)$.

3D Printing of Biomimetic Hydrogels. Cylindrical discs of S-A$\mathrm{CNF}$ and B-A-CNF were 3D printed using an INKREDIBLE 3D bioprinter, CELLINK AB, (Gothenburg, Sweden), which is a pneumatic-based extrusion bioprinter. The solid discs $(10 \mathrm{~mm}$ diameter, $4 \mathrm{~mm}$ height, 10 layers) were designed using the CAD software 123D Design (Autodesk) and the created STL files were subsequently converted into g-code using Repetier-Host (Repetier Server) software. A nozzle diameter of $0.5 \mathrm{~mm}$ was used at a pressure of $5 \mathrm{kPa}$ and a dosing distance of $0.05 \mathrm{~mm}$. The printing parameters were determined from visual printing resolution of grid structures (15 $\times 15 \mathrm{~mm}^{2}$, three layers). The two ink formulations were 3D printed directly onto a glass Petri dish, then cross-linked in a bath of a $90 \mathrm{mM}$ aqueous solution of $\mathrm{CaCl}_{2}$ for 30 min directly on the Petri dish, and finally washed with deionized water.

Chemical Composition. The compositions of the purified stipe and blade were studied in terms of alginate and cellulose content, starting with a dry weight of $10 \mathrm{~g}$. For the isolation of the alginate, the procedure of Zubia et al. was followed using the formaldehyde alkali treatment method. ${ }^{19}$ The precipitate was washed with absolute ethanol followed by acetone, prior to drying for $24 \mathrm{~h}$ at $40{ }^{\circ} \mathrm{C}$. The alginate fraction was expressed as a percentage of dry weight.

The cellulose content was extracted following a modified method described by Siddhanta et al., 2009. ${ }^{20}$ The samples were defatted repeatedly with methanol, placed in a $600 \mathrm{~mL} \mathrm{NaOH}(0.5 \mathrm{M})$ solution at $60{ }^{\circ} \mathrm{C}$ overnight, washed and, dried in room temperature. For the removal of any remaining minerals, the dried material was resuspended in a $200 \mathrm{~mL}$ solution of hydrochloric acid (5\% v/v), washed, and dried for $24 \mathrm{~h}$ at $40{ }^{\circ} \mathrm{C}$. The cellulose fraction was expressed as a percentage on a dry-weight basis.

Optical Microscopy. An optical microscope, Nikon Eclipse LV100N POL (Kanagawa, Japan), and the imaging software NISElements D 4.30 were used to study how the fibrillation process affected the material structure and its micrometer-scale size. Polarized optical microscopy (POM) reference images with a polarization filter were also captured. 
Rheological Properties. Viscosity measurements were performed during the nanofibrillation process to evaluate the degree of fibrillation, and the process was stopped once a viscosity plateau was reached. A Vibro Viscometer SV-10 from A\&D Company, Ltd. (Tokyo, Japan) was used at a constant shear rate and with periodical circulation of the sensor plates from zero to peak (sine-wave vibration) at a frequency of $30 \mathrm{~Hz}$. Because the temperature increased during the nanofibrillation owing to the compression and abrasive shearing forces, the viscosity measurements were repeated at a stabilized temperature of $22.3 \pm 1.0{ }^{\circ} \mathrm{C}$ to confirm that a viscosity plateau had been reached during the process. The presented values are an average of three measurements for each sample.

The rheological behavior of the hybrid inks, S-A-CNF and B-ACNF, was further analyzed using a Discovery HR-2 rheometer from TA Instruments (Elstree, UK) at $25{ }^{\circ} \mathrm{C}$. A cone-plate $(20 \mathrm{~mm}$ ) configuration was used and the shear viscosity was measured at shear rates of $0.01-1000 \mathrm{~s}^{-1}$. Furthermore, the change in moduli while cross-linking the ink was measured with a plate-plate configuration ( $8 \mathrm{~mm}$, gap $500 \mu \mathrm{m}$ ). Oscillation frequency measurements were conducted at $0.1 \%$ strain and a frequency of $1 \mathrm{~Hz}$ for $10 \mathrm{~min}$, using oscillation amplitude sweeps to establish the linear viscoelastic region. $50 \mathrm{~s}$ after the start of the measurement, a $1 \mathrm{~mL}$ drop of a $90 \mathrm{mM}$ $\mathrm{CaCl}_{2}$ solution was added around the inks, causing gelling while the storage and loss moduli were measured simultaneously.

Atomic Force Microscopy. The S-A-CNF and B-A-CNF inks were studied using atomic force microscopy (AFM). The fibrillated sample suspension $(0.01 \mathrm{wt} \%)$ was dispersed and deposited by spin coating onto a clean mica surface for imaging. The measurements were performed with a Veeco Multimode Scanning Probe (Santa Barbara, USA) in tapping mode, with a TESPA tip [antimony- (n-) doped silicon], Bruker (Camarillo, USA). The nanofiber size (width) was measured from the height images using the Nanoscope $\mathrm{V}$ software, and the average values and deviations presented here are based on 50 different measurements. All measurements were conducted in air at room temperature.

Mechanical Properties. The prepared films were tested using conventional tensile testing to provide an indication of the degree of fibrillation. Prior to mechanical testing, the density was calculated using a gravimetric method: an analytic balance was used to determine the weight and a micrometer gauge was used to measure the thickness based on an average of 10 different measurements per material. The sample preparation and the tensile testing procedure have been reported in our previous study. ${ }^{21} \mathrm{~A}$ tensile testing system (AG X; Shimadzu, Japan) equipped with a $1 \mathrm{kN}$ load cell was used at an extension rate of $2 \mathrm{~mm} / \mathrm{min}$ and with a gauge length of $20 \mathrm{~mm}$. All samples were conditioned at $50 \pm 2.0 \%$ relative humidity at $22.1 \pm$ $1.0{ }^{\circ} \mathrm{C}$ for at least $48 \mathrm{~h}$. The results are averages of at least 10 sets of measurements for each material.

Scanning Electron Microscopy. The cross-sections of the stipe and blade raw material were observed using a scanning electron microscopy (SEM) system (JSM-IT300LV, Tokyo, Japan) at an acceleration voltage of $15 \mathrm{kV}$ to study their microstructures. In addition, the cross-sections of the films were observed. All samples were coated using a sputter-coater (EM ACE200, Leica, Wetzlar, Germany) with a gold target to prevent electron charging. The coating was performed in a vacuum of approximately $6 \times 10^{-5} \mathrm{mbar}$ under a current of $100 \mathrm{~mA}$ for $20 \mathrm{~s}$ to obtain a coating thickness of 10 $\mathrm{nm}$.

Compression Properties. Uniaxial unconfined compression tests of the $3 \mathrm{D}$ printed and cross-linked hydrogels were carried out using a dynamic mechanical analyzer (DMA Q800, TA Instruments, New Castle, USA) at $25^{\circ} \mathrm{C}$. The hydrogels were preloaded using a load of $0.05 \mathrm{~N}$ and subsequently compressed up to a strain of $100 \%$ and at a strain rate of $10 \% \mathrm{~min}^{-1}$. The materials were compared according to the stress and tangent modulus at compressive strain levels of 30 and $60 \%$, respectively. The discs were tested six times for each material; the average results are reported.

Stability in Aqueous Media. The mechanical stability of the cross-linked hydrogels was evaluated in aqueous media. Prior to immersion, the samples were frozen at a temperature of approximately
$-20{ }^{\circ} \mathrm{C}$ for at least $24 \mathrm{~h}$; then, they were freeze-dried for $48 \mathrm{~h}$ at a temperature of $-40{ }^{\circ} \mathrm{C}$ using a freeze-dryer (Alpha 1-2 LD plus, Martin Christ Gefriertrocknungsanlagen $\mathrm{GmbH}$, Osterode am Harz, Germany). The swelling ratio was recorded at equilibrium swelling and after 1 week of immersion in 5 wt \% phosphate buffered saline (PBS) solution at $37^{\circ} \mathrm{C}$, according to

$$
\text { swelling ratio }\left(\mathrm{g} \mathrm{g}^{-1}\right)=\left[\left(W_{1}-W_{0}\right) / W_{0}\right]
$$

where $W_{0}$ and $W_{1}$ denote the mass of the sample in dried state and the swollen hydrogel state, respectively. The average results are reported based on measurements of three samples.

X-ray Microtomography. The 3D internal architecture of the natural structures after purification, as well as the reassembled nanostructures after fibrillation, 3D printing, and cross-linking, were reconstructed from the stipe and blade, respectively, after freeze drying of the samples using the conditions described above. Samples of approximate size $64 \mathrm{~mm}^{3}$ were scanned using a Zeiss Xradia 510 Versa (Carl Zeiss, Pleasanton, CA, USA) with a $20 \times$ objective, which performed interior tomography with a field of view of $0.56 \mathrm{~mm}$ and voxel size of $0.56 \mu \mathrm{m}$. The scanned region of interest was positioned at the exact center of each aerogel sample. The scanning was carried out with an X-ray tube voltage of $50 \mathrm{kV}$, an output power of $4 \mathrm{~W}$, and without any X-ray filters. A total of 2401 projections were acquired with an exposure time of $6 \mathrm{~s}$, which resulted in a total scan time of $6 \mathrm{~h}$. The tomographic reconstruction was carried out using filtered backprojection with Zeiss Scout-and-Scan Reconstructor software (version 11.1). The $3 \mathrm{D}$ visualization and analysis of the microstructure in the aerogels were obtained using Dragonfly Pro Software (ORS).

Cytotoxicity Testing of Ink. The impact on cell proliferation of S-A-CNF was tested by culturing fibroblasts in the presence of S-A$\mathrm{CNF}$ ink. Human primary dermal fibroblasts were isolated according to previously described protocols. ${ }^{22}$ Skin was obtained from healthy patients undergoing routine plastic surgery (Swedish Ethical Review Authority no. 2018/97-31). The dermal layer was dissected, cut into 3 $\mathrm{mm}$ square pieces, and incubated at $37{ }^{\circ} \mathrm{C}$ overnight in Dulbecco's modified Eagle's medium (DMEM) containing $2 \%$ fetal calf serum (FCS), $165 \mathrm{U} / \mathrm{mL}$ collagenase (type 1 ; Worthington, Lakewood, N.J., USA) and $2.5 \mathrm{U} / \mathrm{mLdispase}$ (Gibco BRL, Life Technologies, Eggenstein, Germany). Cells were expanded by incubation in DMEM with $10 \%$ FCS in $25 \mathrm{~cm}^{2}$ culture flasks at $37{ }^{\circ} \mathrm{C}, 5 \% \mathrm{CO}_{2}$ and $95 \%$ humidity. Following establishment of primary cultures, cells were subjected to a solution containing $5 \%(\mathrm{w} / \mathrm{v})$ S-A-CNF or PBS. Daily, cells were detached using ethylene-diamine-tetraacetic acid $(0.01 \%) /$ trypsin $(0.125 \%)$ solution at $37{ }^{\circ} \mathrm{C}$ for approximately 10 min, stained with trypan blue to distinguish viable cells, and counted using an EVE Automated Cell Counter (NanoEnTek, Waltham, MA). All experiments were performed in biological triplicates (separate cell cultures) and methodological duplicates (staining and cell counting).

Staining of Proliferative Cells. Cells cultured for two days in the presence of S-A-CNF or PBS were stained using antibodies directed toward the proliferation marker Ki-67. ${ }^{23}$ Briefly, cells were fixated using $4 \%$ neutral buffered paraformaldehyde, rinsed in PBS and incubated with blocking serum (5\% bovine serum albumin) for $1 \mathrm{~h}$. Cells were incubated for $1 \mathrm{~h}$ with $1 \mu \mathrm{g} / \mathrm{mL}$ polyclonal antibodies specific for Ki-67 (ab15580, Abcam Cambridge, UK). After being rinsed in PBS, cells were incubated for $1 \mathrm{~h}$ with Alexa546-conjugated secondary antibodies (dilution 1:500; Molecular Probes, OR), and phalloidin conjugated with Alexa488 (dilution 1:500, ThermoFisher, UK). Finally, cells were rinsed in PBS, stained with 4',6-diamidino-2phenylindole (dilution 1:1000, Sigma-Aldrich) and mounted using Prolong Gold (ThermoFisher, UK). Negative controls included omission of primary antibodies. Stained cells were visualized using laser scanning confocal microscopy (LSM 800, Carl Zeiss AG, Oberkochen, Germany). Ki-67 positive cells were counted and expressed as percent of total number of cells.

Cytotoxicity Testing of Cross-linked Hydrogel. The $\mathrm{CaCl}_{2}$ cross-linked hydrogel from S-A-CNF was also evaluated for in vitro cytotoxicity, performed according to ISO 10993-5:2009, annex C (3(4,5-dimethylthiazol-2-yl)-2-5-diphenyltetrazolium bromide (MTT) 
Table 1. Corresponding Sample Code of Raw Materials and Their Yields, As Well As Their Cellulose and Alginate Content after the Purification

\begin{tabular}{|c|c|c|c|c|c|c|}
\hline raw materials & initial weight $[\mathrm{g}]$ & bleached weight $[\mathrm{g}]$ & total yield $[\%]$ & cellulose $[$ wt $\%]$ & alginate $[\mathrm{wt} \%]$ & sample code \\
\hline stipe & 70 & 50 & $71 \pm 8$ & $33 \pm 6$ & $45 \pm 13$ & S-A-CNF \\
\hline blade & 70 & 52 & $74 \pm 7$ & $23 \pm 3$ & $46 \pm 11$ & B-A-CNF \\
\hline
\end{tabular}

cytotoxicity test). The test system used was a subconfluent monolayer of L929 mouse fibroblast cells (NCTC clone 929: CCL-1 American Type Culture Collection). Positive (Latex rubber, Gammex 91-325, AccuTech Ansell) and negative (Thermanox Plastic Coverslips, Art no 174934, Thermo Scientific NUNC) controls were run in parallel. The data are expressed as the mean \pm standard deviation of six replicates for each material.

The extraction of the test item was performed using culture medium with serum as extraction vehicle: Eagle's minimum essential medium $1 \times$ with nonessential amino acids and sodium pyruvate, supplemented with $5 \%(\mathrm{v} / \mathrm{v})$ fetal bovine serum, $4 \mathrm{mM}$ L-glutamine, $100 \mathrm{IU} / \mathrm{mL}$ penicillin and $100 \mu \mathrm{g} / \mathrm{mL}$ streptomycin. After extraction completion, the extract was checked for changes in $\mathrm{pH}$ and visual signs of particles, before subsequent dilution and addition to cells. Extracts from test item, positive and negative controls as well as blanks (extraction vehicle not containing the test item but subjected to conditions identical to those to which the test item was subjected to during extraction) were added to a subconfluent monolayer of L929 mouse fibroblast cells and incubated for $24 \mathrm{~h}$ at $37 \pm 1{ }^{\circ} \mathrm{C}$ in $5 \%$ $\mathrm{CO}_{2}$. After incubation, the extracts were removed and MTT solution was added to the cells that were incubated for additional $2 \mathrm{~h}$ at $37 \pm 1$ ${ }^{\circ} \mathrm{C}$ in $5 \% \mathrm{CO}_{2}$. Following incubation, the MTT solution was removed, 2 -propanol was added, and the plates were shaken rapidly. Finally, the absorbance was measured at $570 \mathrm{~nm}$ (reference wavelength $650 \mathrm{~nm}$ ) and the viability of cells was calculated. Cell viability was normalized to cells exposed to blank medium and a value above $70 \%$ was considered having noncytotoxic potential.

\section{RESULTS AND DISCUSSION}

Purification and Characterization of Raw Material. The yield and chemical composition after the purification of the raw materials is presented in Table 1.

The objective of the purification of the seaweed was to remove the pigment and other impurities while maintaining as much of the inherently high alginate content in brown seaweed, together with the cellulose content. Indeed, the yield of the stipe and blade were as high as 71 and $74 \%$, respectively, after the bleaching procedure (Table 1); these yields are comparable to that of wood after direct bleaching (namely, about 70\%), which is mainly composed of holocellulose.

An alginate content of $25-30 \mathrm{wt} \%$ and cellulose content of $10-15$ wt \% have previously been reported for raw $L$. digitata seaweed harvested in Scotland during May. ${ }^{24}$ From Table 1, after the purification, the alginate and cellulose contents were higher, but their relative percentages were maintained. The stipe had a higher cellulose content, although both structures showed similar alginate content. However, the standard deviations were high, which might reflect the heterogeneity of the raw material even within a species. ${ }^{25}$

Nanofibrillation Process and Characterization of Hybrid Inks. Viscosity measurements and OM were used to study the degree of fibrillation throughout the process. A plateau in the viscosity curve indicated strong gel formation by the separated nanofibers with a high aspect ratio, and the microscopy measurements confirmed the absence of intact larger structures at the end of the process. The viscosity measured at room temperature as a function of the energy demand is shown in Figure 1 and the route from the raw materials to the hybrid inks is shown in Figure 2.

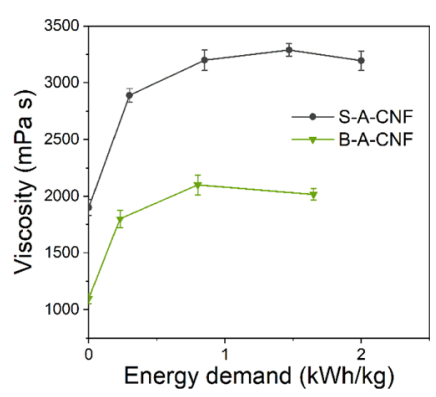

Figure 1. Measured viscosity as a function of the energy consumption during the nanofibrillation process for the preparation of S-A-CNF and B-A-CNF.

An increased viscosity and a plateau are clearly observed for both S-A-CNF and B-A-CNF, as shown in Figure 1. For comparison, commercially bleached kraft wood pulp nanofibrillated with an equivalent technique has a significantly lower viscosity plateau at $1565 \mathrm{mPa} \mathrm{s}{ }^{26}$ In a previous work on the nanofibrillation process for wood pulp, the viscosity was used as an indication of the degree of fibrillation during the process, where the viscosity plateau signified the formation of a strong network of separated nanofibers. ${ }^{26,27}$ Remarkably, the formation of this network of separated cellulosic nanofibers was possible to detect throughout the nanofibrillation process, despite the high amount of noncellulosic components, such as alginate.

A more organized structure was observed for the stipe (Figure 2a) compared with the more layer-like structure of the blade (Figure $2 \mathrm{~b}$ ), which displayed a wide range of cell sizes. The brownish/greenish color of the raw materials (inset Figure $2 a, b)$, was removed from the mainly intact structures after the purification process, which are displayed as translucent white hydrogel structures (inset Figure 2c,d). After purification, thinner layers of the intact structures were visualized under OM (Figure 2c,d). After the nanofibrillation process, no visible larger structures appeared intact from neither OM, nor POM (Figure S1). The nanofibrillation of the stipe reached a maximum viscosity at an energy demand of $1.5 \mathrm{~kW} \mathrm{~h} / \mathrm{kg}$ (Figure 1). In comparison, the blade had a slightly lower energy demand throughout the process, and the maximum viscosity was measured at an energy demand of $1.0 \mathrm{~kW} \mathrm{~h} / \mathrm{kg}$. The slightly higher energy demand of the stipe could be explained by its higher cellulose content (Table 1), which might require more energy to be separated. In addition, the arrangement of cellulose and alginate in the different parts and cell wall structures likely influence the ease of separation. Figure $2 \mathrm{e}, \mathrm{f}$ shows that nanofibers were successfully separated from both the stipe and the blade, appearing as gels after the separation (inset), and their dimensions were on average $7 \pm 3$ and $6 \pm 3 \mathrm{~nm}$, respectively. Their size distribution is provided in Figure S2. The measured energy consumptions were remarkably low for the nanofibrillation of both seaweed 


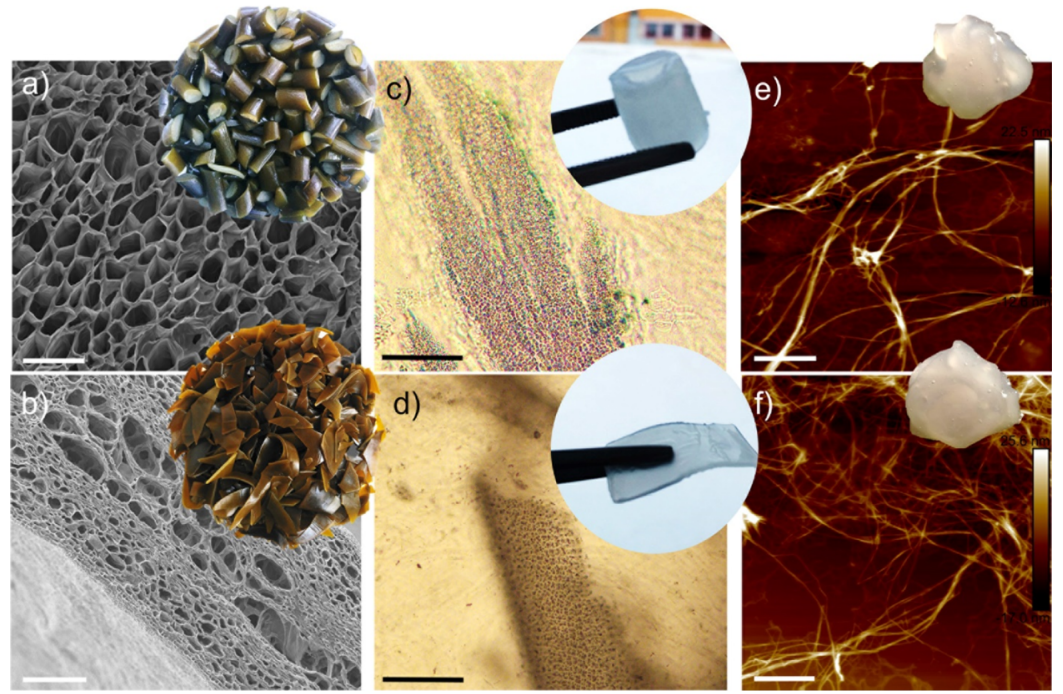

Figure 2. Microstructure of (a) stipe and (b) blade raw material (scale bar: $100 \mu \mathrm{m}$ ); OM after purification of (c) stipe and (d) blade, inset: photographs of the purified stipe and blade, respectively (scale bar: $200 \mu \mathrm{m}$ ); AFM height images of (e) S-A-CNF and (f) B-A-CNF (scale bar: 600 $\mathrm{nm}$ ), inset: photographs of the hybrid inks from stipe and blade, respectively.
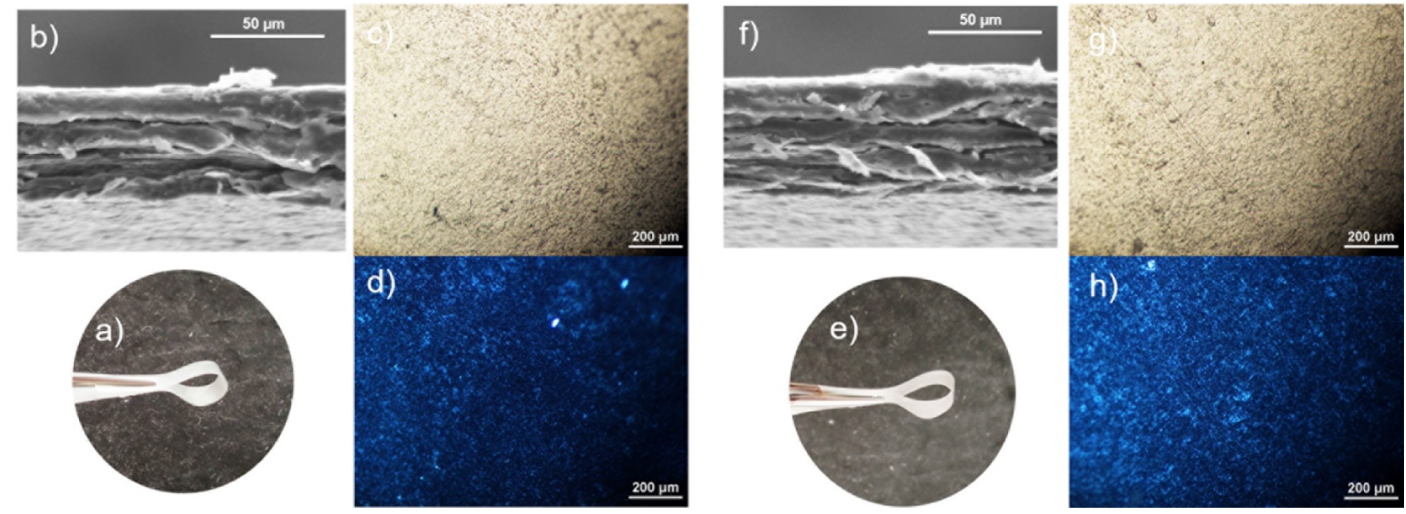

Figure 3. S-A-CNF film: (a) photograph, (b) OM image of surface, (c) POM image of surface, (d) SEM image of cross-section. B-A-CNF film: (e) photograph, (f) SEM image of cross-section, (g) OM image of surface, (h) POM image of surface.

structures and comparable to that of carrot residue ${ }^{21}$ (namely, $0.9 \mathrm{~kW} \mathrm{~h} / \mathrm{kg}$ ); meanwhile, commercially bleached wood kraft pulp reached a maximum viscosity at $8.4 \mathrm{~kW} \mathrm{~h} / \mathrm{kg}$ for a similar processing approach. ${ }^{26}$ The importance of the presence of hemicellulose on the nanofibrillation process of wood pulp have previously been studied using ultrafine grinding. ${ }^{28}$ The low energy demand suggests that the presence of alginate during nanofibrillation may also be beneficial for the separation of nanofibers.

The nanofiber suspensions were further solvent-casted for the preparation of films to evaluate the mechanical properties of the network and to provide an indication of the degree of fibrillation. Photographs and microscopy images of the films are shown in Figure 3.

The films were further characterized in terms of density and mechanical properties, which are listed in Table 2.

\section{Table 2. Properties of the S-A-CNF and B-A-CNF Films}

$\begin{array}{ccccc}\text { films } & \begin{array}{c}\text { E-modulus } \\ {[\mathrm{GPa}]}\end{array} & \begin{array}{c}\text { strength } \\ {[\mathrm{MPa}]}\end{array} & \begin{array}{c}\text { strain at break } \\ {[\%]}\end{array} & \begin{array}{c}\text { density } \\ {\left[\mathrm{g} / \mathrm{cm}^{3}\right]}\end{array} \\ \text { S-A-CNF } & 11.2 \pm 1.2 & 167.5 \pm 9.1 & 2.3 \pm 0.6 & 1.3 \pm 0.4 \\ \text { B-A-CNF } & 9.6 \pm 0.9 & 143.1 \pm 10.0 & 1.7 \pm 0.3 & 1.2 \pm 0.3\end{array}$

Figure 3 shows transparent films (a) with an even surface, where no intact structures or agglomeration could be observed $(b, c, g, h)$. The cross-sections of both films displayed a structure of distinct layers $(\mathrm{d}, \mathrm{f})$. A layered structure is typically found in nanofiber networks; ${ }^{26}$ however, the nanofibers appeared embedded in the alginate and no stacked fibers were visible.

The representative stress-strain curves from the uniaxial tension tests of the films after the nanofibrillation process are provided in the Supporting Information (Figure S3). Both films showed a high modulus and strength, but low strain to break, indicating a successful nanofibrillation, allowing a strong network formation. It has been reported that the addition of CNFs increases the strength and modulus but decreases the strain and flexibility of $\mathrm{CaCl}_{2}$-cross-linked alginate films. ${ }^{29}$ Furthermore, by increasing the CNF content in the films from 1 to $15 \%$, the strength was further increased by $35 \%$. Another study that combined CNF (at 90\%) and alginate (at 10\%) films with $\mathrm{CaCl}_{2}$ cross-linking, reported the formation of an interpenetrating network, which was further locked by ionic cross-linking into a tough material both in the wet and the dry states. ${ }^{30}$ S-A-CNF displayed about $15 \%$ higher E-modulus and $16 \%$ higher strength than B-A-CNF. This could be attributed 

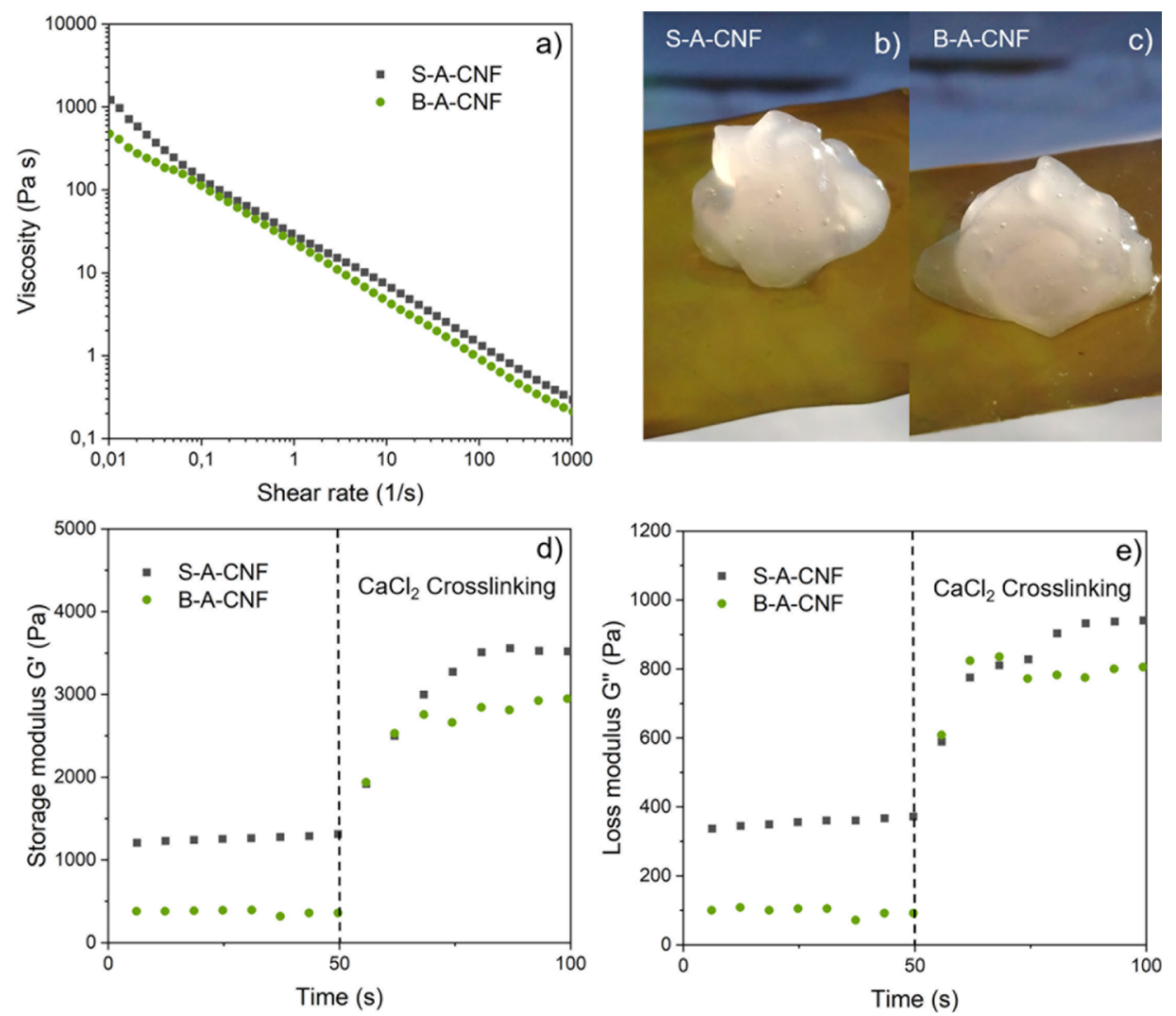

Figure 4. Rheological data of the hybrid inks: (a) viscosity as a function of shear rate, (b,c) photographs of the hybrid inks gels at 2 wt \%, (d) storage modulus $G^{\prime}$, and (e) loss modulus $G^{\prime \prime}$ measured over time, where the $\mathrm{CaCl}_{2}$ solution was added $50 \mathrm{~s}$ after the measurement was started.

to the higher cellulose content of S-A-CNF, thus rendering a higher CNF loading.

The mechanical properties of the s films shown in Table 2 are comparable to those of commercially extracted alginate with added CNF, which has a strength of $100 \mathrm{MPa}$, and a strain of about $1.5 \%$, although with a higher modulus of $17-18$ $\mathrm{GPa}^{29}$ It should be noted that these films were cross-linked with $\mathrm{CaCl}_{2}$, which has been previously shown to significantly enhance the mechanical properties of the films. ${ }^{31}$

3D Printability and Characterization of Biomimetic Hydrogels. The rheological behavior of the hybrid inks was studied to evaluate their suitability for $3 \mathrm{D}$ printing and the results are shown in Figure 4.

In Figure 4a a shear-thinning behavior is observed for both the S-A-CNF and B-A-CNF inks, similar to that seen in viscosity graphs previously reported for commercial alginate mixed with $\mathrm{CNF}^{9}$ and for pure CNF. ${ }^{7}$ For S-A-CNF, the initial viscosity was $1224 \mathrm{~Pa} \mathrm{~s}$ and it decreased to $0.3 \mathrm{~Pa} \mathrm{~s}$ upon increasing the shear rate to $1000 \mathrm{~s}^{-1}$. In comparison, the viscosity of B-A-CNF was initially lower at $578 \mathrm{~Pa} \mathrm{~s}$ and dropped to $0.2 \mathrm{~Pa} \mathrm{~s}$ at a shear rate of $1000 \mathrm{~s}^{-1}$. In addition, the higher viscosity of S-A-CNF can be seen in Figure 4b, which displays a more stable gel formation than that of B-A-CNF, shown in (c). These results are in agreement with the viscosity measurements performed to indicate the degree of fibrillation.

The high viscosity at low shear rates and the shear-thinning behavior with increasing shear rate provide shape fidelity during printing; however, to maintain structural integrity after printing, cross-linking of the alginate is required. Hence, the gelling behavior of the hybrid inks was studied by measuring the loss and storage moduli as a function of time while cross- linking with $\mathrm{CaCl}_{2}$ (Figure 4d,e). Both the storage modulus (d) and the loss modulus (e) displayed an instant increase upon the addition of a $\mathrm{CaCl}_{2}$ solution at $50 \mathrm{~s}$ and became gradually linear after an additional $50 \mathrm{~s}$. The time was measured for an additional $5 \mathrm{~min}$ to confirm this plateau. The higher storage modulus of S-A-CNF reflects a higher degree of cross-linking and results in an increased strength or rigidity.

3D printability and cross-linking enable the use of inks in a wide range of applications that, for example, require specific shapes for wound dressing ${ }^{32}$ or even for 3D printing of living tissues and organs. ${ }^{7}$ The printability and stability of $3 \mathrm{D}$ discs of S-A-CNF and B-A-CNF inks prepared at $2 \mathrm{wt} \%$ solid content were studied, and the printing parameters were tuned through a trial-and-error method according to the visual resolution of the grid structure (Figure S4). The S-A-CNF ink displayed the highest visual resolution and stability upon $3 \mathrm{D}$ printing, yet both hybrid inks could be printed without collapse of the structure (Figure S4). However, a minor shrinkage and some swelling at the center, appearing as a slightly convex surface, were observed after cross-linking of the discs. These tendencies of shape deformation after $\mathrm{CaCl}_{2}$ cross-linking have previously been reported for $3 \mathrm{D}$ printed alginate/CNF hydrogels. ${ }^{7,32}$ This behavior might reflect inadequate homogeneity of the diffusion-based $\mathrm{CaCl}_{2}$ cross-linking approach. Ionic crosslinking of alginate using $\mathrm{CaCl}_{2}$ has been widely studied. By varying parameters such as the cross-linking ratio ${ }^{33}$ and the cross-linking time, ${ }^{34}$ the mechanical properties of printed hydrogels can be tuned. Other factors such as the molecular weight and the $M / G$ ratio are related to the raw material and its alginate extraction process. These factors have a large influence both on the cross-linking behavior and the 

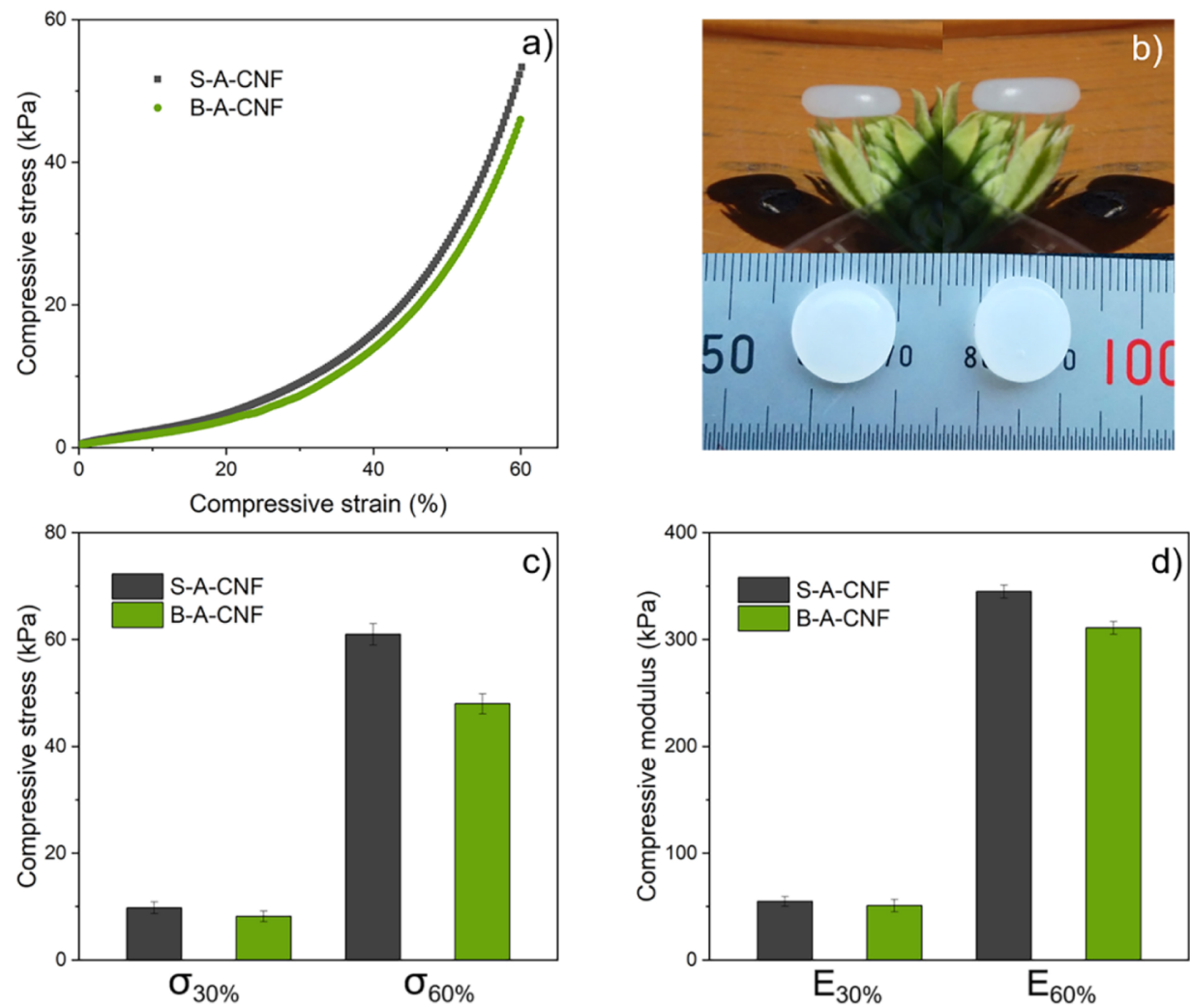

Figure 5. (a) Representative compressive stress-strain curves for a strain up to 60\%, (b) photographs of the hydrogels after cross-linking, (c) compressive strength, and (d) compressive modulus at 30 and $60 \%$ strain.

fundamental mechanical behavior of the hydrogels. ${ }^{5}$ The $3 \mathrm{D}$ printed S-A-CNF and B-A-CNF hydrogels were evaluated under compression to determine their mechanical properties after cross-linking, as shown in Figure 5.

Because the compressive stress and strain curves revealed a viscoelastic nonlinear stress-strain behavior, the compressive modulus and strength at 30 and $60 \%$ strain were used for mechanical characterization (Figure $5 \mathrm{a}$ ) of the $3 \mathrm{D}$ printed hydrogels (b). In Figure 5c,d, S-A-CNF shows generally better compressive properties than B-A-CNF. This is in good agreement with the rheological behavior of the gels and could be explained by the higher amount of CNF in S-A-CNF reinforcing the structure. However, the stiffness of alginate hydrogels is directly related to their cross-linking, and S-A$\mathrm{CNF}$ with a relatively lower amount of alginate than B-A-CNF; displayed a higher stiffness, as seen In Figure $5 \mathrm{~d}$.

In L. digitata, a higher amount of alginate rich in guluronic acid (G), instead of mannuronic acid (M), was previously shown for the stipe than that of the blade of the seaweed; ${ }^{6}$ therefore, the stipe has a lower $M / G$ ratio. Alginates with lower $\mathrm{M} / \mathrm{G}$ ratio are known to display a higher affinity to crosslinking (mechanical rigidity), and the gel strength of the alginate is mainly dependent on the content and length of $\mathrm{G}^{35}$ A lower $M / G$ ratio of the alginate in the S-A-CNF hydrogel than that of B-A-CNF may further contribute to the higher compressive properties of the former, in particular, its stiffness.

It is also notable that the maximum compressive strength was measured at around $80 \%$ strain for the B-A-CNF hydrogel $(175.2 \pm 3 \mathrm{kPa}$ ), shown in the compressive graphs (Figure $\mathrm{S} 5 \mathrm{a})$. At this strain, the B-A-CNF hydrogel fractured while the S-A-CNF hydrogel was only compressed, without any visual fractures (Figure S5b). The natural composition of S-A-CNF assembled into a biomimetic hydrogel with high compressive stiffness and strength, but highly flexible, displaying the contribution from increased CNF content and its favorable contribution on the structural stability. The utilization of the natural composition in this study limits the tuning of the mechanical properties of the hydrogels by altering the ratio of the two components, alginate and CNF, and establishing their separate contributions.

However, the influence of factors such as the concentration of the solid content and $\mathrm{CaCl}_{2}$ has previously been shown to be highly effective for tailoring the mechanical behavior of the hydrogels, ${ }^{36}$ as well as its long-term stability and performance. ${ }^{37}$ Commercial alginate with a dry content of $2 \mathrm{wt} \%$, cross-linked with a $50 \mathrm{mM} \mathrm{CaCl} 2$ solution for hydrogel formation (compared to $90 \mathrm{mM}$ used in this study), displayed a compressive strength and modulus of 25 and $32 \mathrm{kPa}$, respectively, at $60 \%$ strain. ${ }^{36}$ Moreover, it was shown that these values could be improved to some extent by increasing the solid content and $\mathrm{CaCl}_{2}$ concentration. In Figure $5 \mathrm{c}, \mathrm{d}$, the strength and modulus of the S-A-CNF hydrogel are 61 and 345 , respectively, at the same strain, signifying a contribution from entangled CNF network. At 30\% strain, the S-A-CNF hydrogel displayed mechanical properties (Figure 5c,d), comparable to those of commercial alginate with the addition of 90 wt \% CNF, and at 2.5 wt \% solid content, namely a strength of about $24 \mathrm{kPa}$, and modulus of $65 \mathrm{kPa}^{7}$ The longterm mechanical stability of ionically cross-linked alginate in aqueous media is a challenge that can limit their application in tissue engineering. ${ }^{37}$ Therefore, the hydrogels were immersed in PBS solution at $37^{\circ} \mathrm{C}$ and monitored until the equilibrium swelling ratio was reached. The measurement was then repeated after 1 week of immersion to evaluate the structural 
a)
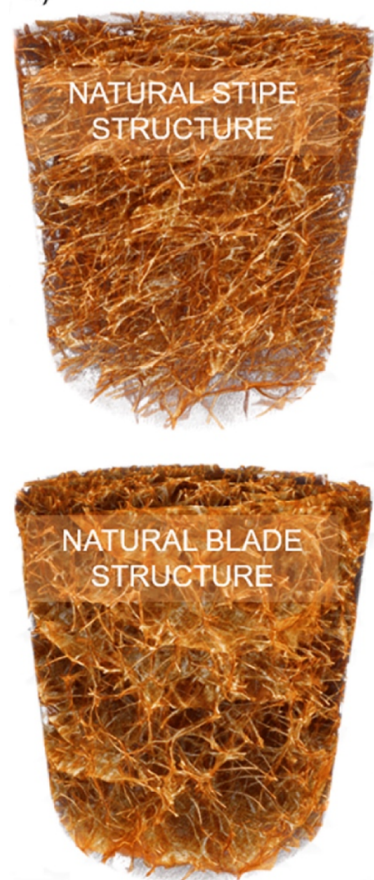

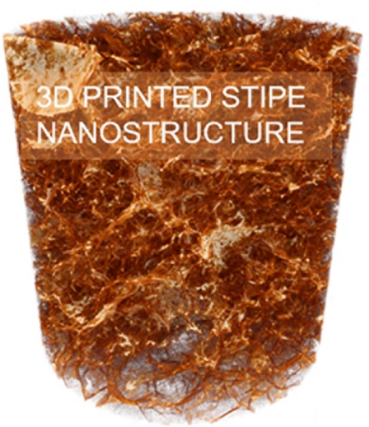

b)

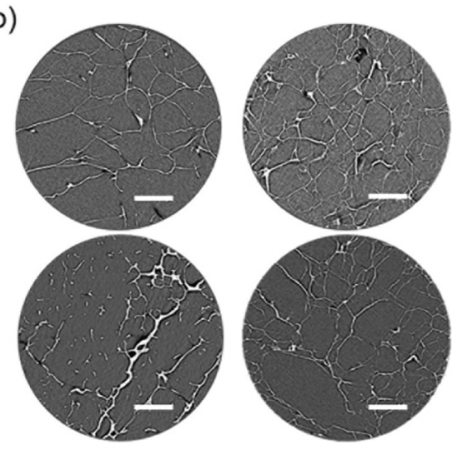

c)

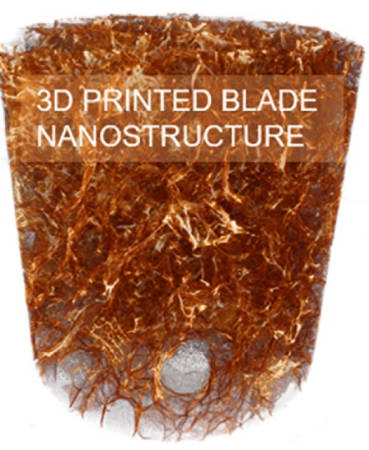

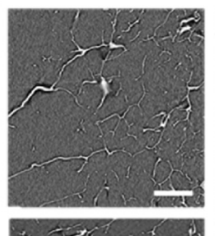
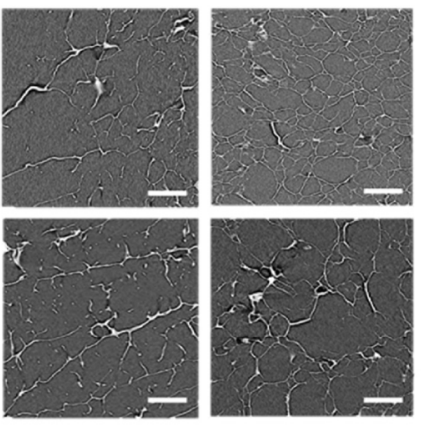

Figure 6. (a) X-ray microtomography 3D reconstruction of the structure of natural seaweed after purification and of the nanofibrillated, 3D printed, and cross-linked structures derived from the seaweed stipe and blade. (b) Horizontal slice of the 3D reconstruction (circular) and (c) longitudinal slice of the $3 \mathrm{D}$ reconstruction (square). The scale bars correspond to $100 \mu \mathrm{m}$.
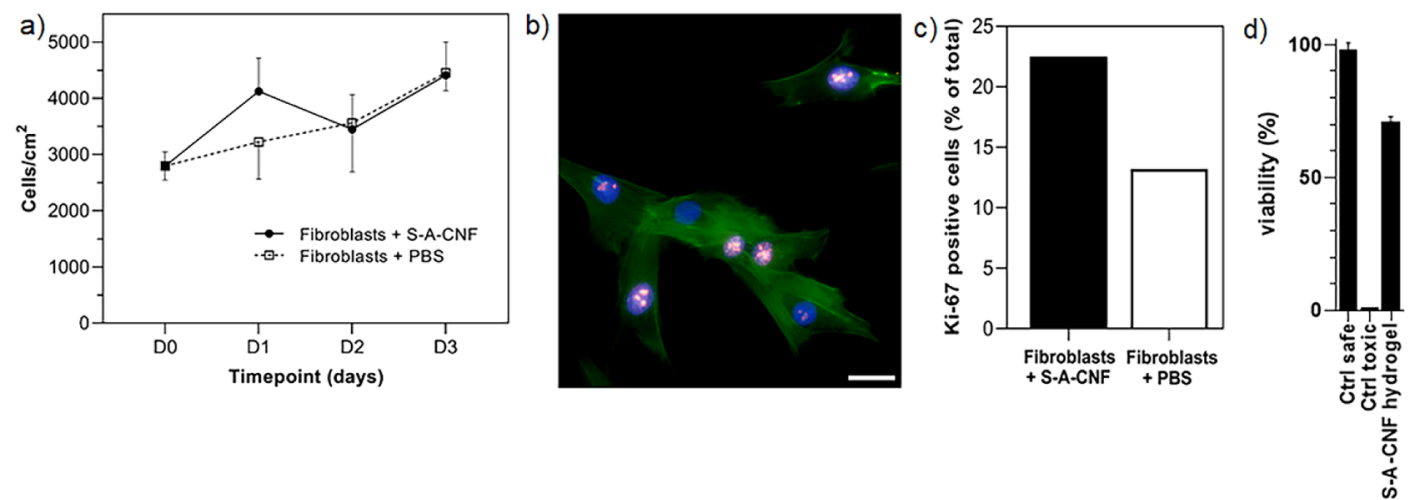

Figure 7. (a) Cytotoxicity tests of S-A-CNF (b) quantification of the number of proliferative cells following exposure to S-A-CNF or PBS, the presence of Ki-67 (red) in the cell nuclei (blue) visualized using immunohistochemistry. F-actin was stained to assess the general cell morphology (green). Scale bar equals $20 \mu \mathrm{m}$. (c) Percentage of cells identified to be in a proliferative phase when cultured in the presence of S-A-CNF compared to PBS control. (d) L929-fibroblast cell viability of the S-A-CNF 3D-printed and cross-linked hydrogel, the negative control sample (Ctrl safe), and the positive control sample (Ctrl toxic).

integrity of the cross-linked hydrogels, and the results are shown in Table S2. After 1 week, there was no weight loss observed for the hydrogels (Table S2), signifying their structural integrity and stability. Furthermore, their swelling ratios are comparable to values reported for $\mathrm{Ca}^{2+}$ cross-linked alginate/CNF hydrogel combinations in $\mathrm{PBS}^{14}$

Long-term stability of alginate cross-linking with $\mathrm{Ca}^{2+}$ was reported to be highly dependent of calcium content and crosslinking of G-blocks that upon forming the "egg-box" conformation, are less susceptible to decross-linking. Also, TEMPO-oxidized CNF have shown to play a crucial role in improving the dimensional stability of the samples when compared to pure alginate gels. ${ }^{14}$

Altogether, the compressive properties imply that the natural composition of the hybrid inks has an inherently favorable mechanical behavior and cross-linking ability, which could be further explored and utilized to adjust its mechanical properties, where the presence of nanofiber further contribute to its long-term performance.

To study the morphology of the natural seaweed structure after purification in comparison with the nanofibrillated and reassembled biomimetic hydrogel structure after cross-linking, the samples were imaged using X-ray microtomography, as shown in Figure 6.

The addition of CNFs has been reported to contribute to the formation of a more favorable structure for cell growth by favoring a web-like structure with larger pores ranging from 40 to $150 \mu \mathrm{m} .{ }^{14}$ As observed in the $3 \mathrm{D}$ reconstruction of Figure $6 \mathrm{a}$, as well as in the $2 \mathrm{D}$ slices (Figure $6 \mathrm{~b}, \mathrm{c}$ ), the nanofibrillation of the cellulosic part of the seaweed into CNFs promoted the 
formation of an interconnected network structure upon reassembling, compared to that of the natural structure.

The natural structures were intact in the form of natural hydrogels after the purification, as shown in Figure 2e,f. After freeze-drying, both the stipe and the blade displayed a porous structure, where the layered structure of the raw blade material observed by SEM (Figure 2b) was apparent also after the purification (Figure 6a-c). The 3D printed hydrogels displayed open interconnected pores with a wide range of pore diameters of $10-150$ and $20-200 \mu \mathrm{m}$ for the stipe and the blade, respectively.

To evaluate the potential use of the developed hybrid inks for biomedical applications such as tissue engineering, the S-ACNF ink having the most promising rheological behavior, was investigated for any potentially harmful effects of the cell viability, both as an ink and after printing and cross-linking into a hydrogel shown in Figure 7.

To assess the cytocompatibility of S-A-CNF ink, its impact on the proliferation of human dermal fibroblasts was tested. Cells were cultured in the presence of 5\% S-A-CNF (weight/ volume) for three days (Figure $7 a-c$ ).

The cytotoxicity tests (Figure $7 \mathrm{a}$ ) revealed no negative or deleterious effect on human fibroblasts in regard to proliferation when compared to control (PBS). Moreover, cells subjected to S-A-CNF or PBS control were stained for the proliferative marker $\mathrm{Ki}-67$, to determine the number of proliferative cells (Figure $7 \mathrm{~b}$ ). Culture with S-A-CNF resulted in $22.5 \% \mathrm{Ki}-67$ positive cells, compared to $13.2 \%$ for the control (Figure $7 \mathrm{c}$ ). Thus, a higher percentage of cells were identified to be in a proliferative phase when cultured in the presence of S-A-CNF compared to PBS control, signifying the potential of the ink to be used for 3D printing of hydrogels for biomedical uses.

The cytotoxicity of the S-A-CNF ink was also evaluated after $3 \mathrm{D}$ printing and $\mathrm{CaCl}_{2}$ cross-linking into a hydrogel (Figure $7 \mathrm{~d})$. The cytotoxicity test of the hydrogel on L929 mouse fibroblast cells showed a cell viability of $71 \pm 2 \%$, classified as noncytotoxic, where the extract has a cytotoxic potential given that the viability is reduced to $<70 \%$. However, there was a notable difference between the negative control $(98 \pm 3 \%)$ and S-A-CNF ink. Moreover, the positive control resulted in complete cell death $(1 \pm 0 \%)$. For indirect assays, the extracts are used to evaluate the effects of possible cytotoxic contaminants that may readily be extracted from the biomaterials. Previous indirect assessment on the cell viability of gel formulations of commercial alginate and $\mathrm{CNF}$, isolated with different processes, have reported values of about $75 \%{ }^{14}$ and $106 \%{ }^{7}$ for addition of 50 , and $90 \mathrm{wt} \% \mathrm{CNFs}$, respectively. It therefore seems reasonable to suggest that the cellulose and alginate source, preparation procedures, sterility and chemicals used have direct influence on the physicochemical properties of the final products, thus affecting their cytotoxicity.

For S-A-CNF hydrogels, the principal contaminants present in the raw material; brown seaweed consist of proteins, endotoxins and polyphenols. ${ }^{38}$ Despite extensive purification of alginates it was shown that relatively high residual amounts of protein contaminants still were present, ${ }^{38}$ which could plausibly affect cell viability. Altogether, the potential for future development and use of the hybrid inks in 3D printing of, for example, soft tissue has been demonstrated.

\section{CONCLUSIONS}

This paper reports on the utilization of the natural composition of brown seaweed to prepare alginate/CNF hybrid inks with an excellent shear-thinning behavior suitable for 3D printing.

It was found that the natural composition of alginate was beneficial for an energy-efficient nanofibrillation process, resulting in very fine nanofibers on average measured to 6 and $7( \pm 3) \mathrm{nm}$ for S-A-CNF and B-A-CNF, respectively. The $\mathrm{S}-\mathrm{A}-\mathrm{CNF}$ ink had a higher $\mathrm{CNF} /$ alginate ratio than B-A-CNF and demonstrated a higher viscosity and better compressive properties of the hydrogels after $\mathrm{CaCl}_{2}$ cross-linking.

Our results demonstrate the potential use of a direct, resource-efficient, and upscalable approach for the preparation of alginate/CNF hybrid inks instead of employing separate methods to extract and combine the cross-linking ability of the alginate with the shear-thinning behavior of the CNF. We conclude that the hybrid inks are suitable for the $3 \mathrm{D}$ printing of hydrogels with a favorable mechanical behavior and stability after cross-linking and an open interconnected network structure. Cytotoxicity testing of S-A-CNF ink revealed no deleterious effects on cell proliferation of human cells, and the cross-linked hydrogels displayed a cell viability of $71 \%$, suggesting potential for future development and use of the inks in $3 \mathrm{D}$ printing of hydrogels for soft tissue engineering.

\section{ASSOCIATED CONTENT}

\section{s1 Supporting Information}

The Supporting Information is available free of charge at https://pubs.acs.org/doi/10.1021/acsabm.0c00920.

Raw material content; OM, POM, and photographs of the materials after purification and after fibrillation; nanofiber size distribution of the hybrid inks; photograph of $3 \mathrm{D}$ printed grid structure before and after crosslinking; stress and strain curves from tensile testing of films; compressive stress and strain curves from testing of the 3D-printed and $\mathrm{CaCl}_{2}$-cross-linked hydrogels and photographs of the hydrogel samples after the compression test; and swelling ratio at equilibrium and after 1 week in PBS solution (PDF)

Videos of the $3 \mathrm{D}$ porous structure of: stipe natural structure (Video S1) (AVI)

stipe nanostructure (Video S2) (AVI)

blade natural structure (Video S3) (AVI)

blade nanostructure (Video S4) (AVI)

\section{AUTHOR INFORMATION}

\section{Corresponding Authors}

Linn Berglund - Division of Materials Science, Department of Engineering Sciences and Mathematics, Luleå University of Technology, SE-971 87 Luleå, Sweden; 이잉.org/00000002-6247-5963; Email: linn.berglund@ltu.se

Kristiina Oksman - Division of Materials Science, Department of Engineering Sciences and Mathematics, Luleå University of Technology, SE-971 87 Luleå, Sweden; Fibre and Particle Engineering, University of Oulu, FI-90014 Oulu, Finland; Mechanical \& Industrial Engineering (MIE), University of Toronto, M5S 3G8 Toronto, Canada; 이이. ord.o0000003-4762-2854; Email: kristiina.oksman@ltu.se

\section{Authors}

Jonathan Rakar - The Center for Disaster Medicine and Traumatology, and Experimental Plastic Surgery, Department 
of Biomedical and Clinical Sciences, Linköping University, SE58183 Linköping, Sweden

Johan P. E. Junker - The Center for Disaster Medicine and Traumatology, and Experimental Plastic Surgery, Department of Biomedical and Clinical Sciences, Linköping University, SE58183 Linköping, Sweden

Fredrik Forsberg - Division of Fluid and Experimental

Mechanics, Department of Engineering Sciences and

Mathematics, Luleå University of Technology, SE-971 87 Luleå, Sweden

Complete contact information is available at:

https://pubs.acs.org/10.1021/acsabm.0c00920

\section{Funding}

Swedish Foundation for Strategic Research within the HEALiX project [RMX18-0039]; the European Regional Development Fund under the Interreg Nord within the Sea-Surf-Snow project [20201287]; Bio4Energy-a strategic research environment, appointed by the Swedish government and Kempe Foundations for the use of AFM and X-ray microtomography.

\section{Notes}

The authors declare no competing financial interest.

\section{ACKNOWLEDGMENTS}

The authors acknowledge the financial support of the Swedish Foundation for Strategic Research, Interreg Nord, Bio4Energy, and Kempe Foundations and Lucas Gerardin and Boris Valldecabres for their assistance on the preparation and testing of the films.

\section{REFERENCES}

(1) FAO, FAO Yearbook, Fishery and Aquaculture Statistics 2017; Food and Agriculture Organization of the United Nations: Rome, 2019.

(2) Misurcova, L. In Handbook of Marine Macroalgae: Biotechnology and Applied Phycology, 1st ed.; Se-Kwon, K., Ed.; JohnWiley \& Sons, Ltd.: New Delhi, India, 2012; pp 181-182.

(3) Manns, D.; Nielsen, M. M.; Bruhn, A.; Saake, B.; Meyer, A. S. Compositional variations of brown seaweeds Laminaria digitata and Saccharina latissima in Danish waters. J. Appl. Phycol. 2017, 29, 14931506.

(4) Black, W. A. P. The seasonal variation in the cellulose content of the common Scottish Laminariaceae and Fucaceae. J. Mar. Biol. Assoc. U. K. 1950, 29, 379-387.

(5) Abdul Khalil, H. P. S.; Lai, T. K.; Tye, Y. Y.; Rizal, S.; Chong, E. W. N.; Yap, S. W.; Hamzah, A. A.; Nurul Fazita, M. R.; Paridah, M. T. A review of extractions of seaweed hydrocolloids: properties and applications. eXPRESS Polym. Lett. 2018, 12, 296-317.

(6) Peteiro, C. In Alginates and Their Biomedical Applications; Rehm, B., Moradali, M., Eds.; Springer Series in Biomaterials Science and Engineering; Springer: Singapore, 2017; Vol. 11, pp 27-58.

(7) Markstedt, K.; Mantas, A.; Tournier, I.; Martínez Ávila, H.; Hägg, D.; Gatenholm, P. 3D Bioprinting Human Chondrocytes with Nanocellulose-Alginate Bioink for Cartilage Tissue Engineering Applications. Biomacromolecules 2015, 16, 1489-1496.

(8) Wang, N.; Yu, K.-K.; Shan, Y.-M.; Li, K.; Tian, J.; Yu, X.-Q.; Wei, $\mathrm{X}$. $\mathrm{HClO} / \mathrm{ClO}$-Indicative Interpenetrating Polymer Network Hydrogels as Intelligent Bioactive Materials for Wound Healing. ACS Appl. Bio Mater. 2020, 3, 37-44.

(9) Abouzeid, R. E.; Khiari, R.; Beneventi, D.; Dufresne, A. Biomimetic mineralization of three-dimensional printed alginate/ TEMPO-oxidized cellulose nanofibril scaffolds for bone tissue engineering. Biomacromolecules 2018, 19, 4442-4452.

(10) Wu, T.; Yu, S.; Lin, D.; Wu, Z.; Xu, J.; Zhang, J.; Ding, Z.; Miao, Y.; Liu, T.; Chen, T.; Cai, X. Preparation, characterization, and release behavior of doxorubicin hydrochloride from dual cross-linked chitosan/alginate hydrogel beads. ACS Appl. Bio Mater. 2020, 3, 3057-3065.

(11) Kong, H.-J.; Lee, K. Y.; Mooney, D. J. Decoupling the dependence of rheological/mechanical properties of hydrogels from solids concentration. Polymer 2002, 43, 6239-6246.

(12) Chinga-Carrasco, G. Potential and limitations of nanocelluloses as components in biocomposite inks for three-dimensional bioprinting and for biomedical devices. Biomacromolecules 2018, 19, 701-711.

(13) Falsini, S.; Bardi, U.; Abou-Hassan, A.; Ristori, S. Sustainable strategies for large-scale nanotechnology manufacturing in the biomedical field. Green Chem. 2018, 20, 3897-3907.

(14) Siqueira, P.; Siqueira, É.; de Lima, A. E.; Siqueira, G.; PinzónGarcia, A. D.; Lopes, A. P.; Segura, M. E. C.; Isaac, A.; Vargas Pereira, F.; Botaro, V. R. Three-dimensional stable alginate-nanocellulose gels for biomedical applications: towards tunable mechanical properties and cell growing. Nanomaterials 2019, 9, 78-100.

(15) Sadhukhan, J.; Gadkari, S.; Martinez-Hernandez, E.; Ng, K. S.; Shemfe, M.; Torres-Garcia, E.; Lynch, J. Novel macroalgae (seaweed) biorefinery systems for integrated chemical, protein, salt, nutrient and mineral extractions and environmental protection by green synthesis and life cycle sustainability assessments. Green Chem. 2019, 21, 26352655.

(16) Nascimento, D. M.; Nunes, Y. L.; Figueirêdo, M. C. B.; de Azeredo, H. M. C.; Aouada, F. A.; Feitosa, J. P. A.; Rosa, M. F.; Dufresne, A. Nanocellulose nanocomposite hydrogels: technological and environmental issues. Green Chem. 2018, 20, 2428-2448.

(17) Jessop, Z. M.; Al-Sabah, A.; Gao, N.; Kyle, S.; Thomas, B.; Badiei, N.; Hawkins, K.; Whitaker, I. S. Printability of pulp derived crystal, fibril and blend nanocellulose-alginate bioinks for extrusion 3D bioprinting. Biofabrication 2019, 11, 045006.

(18) Zhang, X.; Morits, M.; Jonkergouw, C.; Ora, A.; Valle-Delgado, J. J.; Farooq, M.; Ajdary, R.; Huan, S.; Linder, M.; Rojas, O.; Sipponen, M. H.; Österberg, M. Three-Dimensional Printed Cell Culture Model Based on Spherical Colloidal Lignin Particles and Cellulose Nanofibril-Alginate Hydrogel. Biomacromolecules 2020, 21, 1875-1885.

(19) Zubia, M.; Payri, C.; Deslandes, E. Alginate, mannitol, phenolic compounds and biological activities of two range-extending brown algae, Sargassum mangarevense and Turbinaria ornata (Phaeophyta: Fucales), from Tahiti (French Polynesia). J. Appl. Phycol. 2008, 20, $1033-1043$.

(20) Siddhanta, A. K.; Prasad, K.; Meena, R.; Prasad, G.; Mehta, G. K.; Chhatbar, M. U.; Oza, M. D.; Kumar, S.; Sanandiya, N. D. Profiling of cellulose content in Indian seaweed species. Bioresour. Technol. 2009, 100, 6669-6673.

(21) Berglund, L.; Noël, M.; Aitomäki, Y.; Öman, T.; Oksman, K. Production potential of cellulose nanofibers from industrial residues: efficiency and nanofiber characteristics. Ind. Crops Prod. 2016, 92, 84-92.

(22) Junker, J. P. E.; Sommar, P.; Skog, M.; Johnson, H.; Kratz, G. Adipogenic, chondrogenic and osteogenic differentiation of clonally derived human dermal fibroblasts. Cells Tissues Organs 2009, 191, 105-118.

(23) Sun, X.; Kaufman, P. D. Ki-67: more than a proliferation marker. Chromosoma 2018, 127, 175-186.

(24) Schiener, P.; Black, K. D.; Stanley, M. S.; Green, D. H. The seasonal variation in the chemical composition of the kelp species Laminaria digitata, Laminaria hyperborea, Saccharina latissima and Alaria esculenta. J. Appl. Phycol. 2015, 27, 363-373.

(25) Manns, D.; Deutschle, A. L.; Saake, B.; Meyer, A. S. Methodology for quantitative determination of the carbohydrate composition of brown seaweeds (Laminariaceae). RSC Adv. 2014, 4, 25736-25746.

(26) Berglund, L.; Anugwom, I.; Hedenström, M.; Aitomäki, Y.; Mikkola, J.-P.; Oksman, K. Switchable ionic liquids enable efficient nanofibrillation of wood pulp. Cellulose 2017, 24, 3265-3279. 
(27) Lahtinen, P.; Liukkonen, S.; Pere, J.; Sneck, A.; Kangas, H. A comparative study of fibrillated fibers from different mechanical and chemical pulps. Bioresources 2014, 9, 2115-2127.

(28) Iwamoto, S.; Abe, K.; Yano, H. The effect of hemicelluloses on wood pulp nanofibrillation and nanofiber network characteristics. Biomacromolecules 2008, 9, 1022-1026.

(29) Sirviö, J. A.; Kolehmainen, A.; Liimatainen, H.; Niinimäki, J.; Hormi, O. E. O. Biocomposite cellulose-alginate films: Promising packaging materials. Food Chem. 2014, 151, 343-351.

(30) Benselfelt, T.; Engström, J.; Wågberg, L. Supramolecular double networks of cellulose nanofibrils and algal polysaccharides with excellent wet mechanical properties. Green Chem. 2018, 20, $2558-2570$.

(31) Liling, G.; Di, Z.; Jiachao, X.; Xin, G.; Xiaoting, F.; Qing, Z. Effects of ionic crosslinking on physical and mechanical properties of alginate mulching films. Carbohydr. Polym. 2016, 136, 259-265.

(32) Leppiniemi, J.; Lahtinen, P.; Paajanen, A.; Mahlberg, R.; MetsäKortelainen, S.; Pinomaa, T.; Pajari, H.; Vikholm-Lundin, I.; Pursula, P.; Hytönen, V. P. 3D-Printable Bioactivated Nanocellulose-Alginate Hydrogels. ACS Appl. Mater. Interfaces 2017, 9, 21959-21970.

(33) Freeman, F. E.; Kelly, D. J. Tuning alginate bioink stiffness and composition for controlled growth factor delivery and to spatially direct MSC fate within bioprinted tissues. Sci. Rep. 2017, 7, 1-12.

(34) Giuseppe, M. D.; Law, N.; Webb, B.; Macrae, R. A.; Liew, L. J.; Sercombe, T. B.; Dilley, R. J.; Doyle, B. J. Mechanical behaviour of alginate-gelatin hydrogels for 3D bioprinting. J. Mech. Behav. Biomed. Mater. 2018, 79, 150-157.

(35) Lee, K. Y.; Mooney, D. J. Alginate: properties and biomedical applications. Prog. Polym. Sci. 2012, 37, 106-126.

(36) Nunamaker, E. A.; Otto, K. J.; Kipke, D. R. Investigation of the material properties of alginate for the development of hydrogel repair of dura mater. J. Mech. Behav. Biomed. Mater. 2011, 4, 16-33.

(37) Kuo, C. K.; Ma, P. X. Maintaining dimensions and mechanical properties of ionically crosslinked alginate hydrogel scaffolds in vitro. J. Biomed. Mater. Res., Part A 2007, 84, 899-907.

(38) Dusseault, J.; Tam, S. K.; Ménard, M.; Polizu, S.; Jourdan, G.; Yahia, L. H.; Hallé, J.-P. Evaluation of alginate purification methods: effect on polyphenol, endotoxin, and protein contamination. J. Biomed. Mater. Res., Part A 2006, 76, 243-251. 